\title{
p53 mediates apoptosis induced by c-Myc activation in hypoxic or gamma irradiated fibroblasts
}

\author{
Brent A. Rupnow ${ }^{1}$, Rodolfo M. Alarcon ${ }^{1}$, Amato J. Giaccia ${ }^{1}$ \\ and Susan J. Knox ${ }^{1,2}$ \\ ${ }^{1}$ Department of Radiation Oncology, Stanford University School of Medicine, \\ Stanford, California 94305-5302, USA \\ 2 corresponding author: Susan J. Knox, PhD, MD, Stanford University Medical \\ Center, Department of Radiation Oncology, Stanford, CA 94305-5302. \\ tel: (415)725-4796; fax: (415)725-8231
}

Received 29.1.97; revised 22.8.97; accepted 22.9.97

Edited by C.J. Thiele

\begin{abstract}
Deregulated c-Myc expression leads to a cellular state where proliferation and apoptosis are equally favored depending on the cellular microenvironment. Since the apoptotic sensitivity of many cells is influenced by the status of the p53 tumor suppressor gene, we investigated whether the induction of apoptosis by DNA damage or non-genotoxic stress are also influenced by the $\mathrm{p} 53$ status of cells with altered c-Myc activity. Rat-1 fibroblasts expressing a conditional c-Myc allele (cMycER), were transfected to express an antisense RNA complimentary to p53 mRNA. Expression of antisense p53 RNA decreased p53 protein levels and delayed p53 accumulation following c-Myc activation. Under hypoxic or low serum conditions, cells expressing antisense p53 were substantially more resistant to c-Myc-induced apoptosis than were control cells. c-Myc activation also sensitized Rat-1 cells to radiationinduced apoptosis. Rat-1 cells expressing antisense p53 RNA were more resistant to apoptosis induced by the combined effects of c-Myc activation and gamma irradiation. In a similar manner, apoptosis induced by c-Myc in serum starved, hypoxic or gamma irradiated fibroblasts was also inhibited by $\mathrm{Bcl}-2$. These data indicate that p53 is involved in c-Mycmediated apoptosis under a variety of stresses which may influence tumor growth, evolution and response to therapy.
\end{abstract}

Keywords: c-Myc; p53; apoptosis; hypoxia; radiation

Abbreviations: c-MycER, c-Myc-estrogen receptor fusion protein; 4HT, 4 Hydroxytamoxifen; PI, propidium iodide; IGF1, insulin-like growth factor-1; PDGF, platelet derived growth factor; EGF, epidermal growth factor; bFGF, basic fibroblast growth factor; DMEM, Dulbecco's modified Eagles media; FBS, fetal bovine serum

\section{Introduction}

Alterations in the c-Myc proto-oncogene and the p53 tumor suppressor gene are among the most common genetic abnormalities detected in human cancers. c-Myc acts as a transcriptional regulator of a diverse group of genes which are necessary for cell cycle progression and are involved in cellular differentiation and transformation (Meichle et al, 1992). In addition to its role as a positive regulator of cellular proliferation, activation of c-Myc can lead to apoptosis under certain environmental conditions including growth factor or oxygen deprivation (Evan et al, 1992; Graeber et al, 1996). cMyc-induced apoptosis under these conditions can be inhibited by overexpression of the Bcl-2 protein (Bissonnette et al, 1992; Fanidi et al, 1992; Graeber et al, 1996). Bcl-2 is a member of an extensive family of proteins which regulate cell death induced by a variety of signals. Interestingly, c-Myc and $\mathrm{Bcl}-2$ have been shown to cooperate in cellular transformation and tumorigenesis (Strasser et al, 1990). This observation suggests that inhibition of c-Myc-induced apoptosis might be an important step in the generation and progression of tumors from cells containing altered c-Myc. However, c-Myc activation in cells under normal growth conditions results in proliferation rather than apoptosis, which only occurs when a second environmental stress such as hypoxia or growth factor deprivation is present (Evan et al, 1992; Graeber et al, 1996).

Recent evidence has suggested that components of the tumor microenvironment may provide the stress necessary to induce apoptosis of transformed cells in vivo (Graeber et al, 1996). Mouse tumors derived from fibroblasts transformed by expression of the adenovirus E1A and the H-ras oncogenes exhibited extensive apoptosis which occurred specifically in regions of tumor hypoxia, a commonly occurring condition found in solid tumors. Apoptosis of hypoxic cells in these tumors was found to be dependent on the presence of the wild-type p53 tumor suppressor gene. The protein product of the p53 gene can act as a transcriptional regulator of $\mathrm{G} 1$ cell cycle arrest in response to DNA damage and as an important mediator of apoptosis induced by a variety of stimuli (Lowe, 1995).

Recent studies have suggested that p53 might play an important role in c-Myc-induced apoptosis of serum starved fibroblasts (Hermeking and Eick, 1994; Wagner et al, 1994). Also, we have recently demonstrated that activation of cMyc in an animal tumor model resulted in apoptosis of cells residing in hypoxic regions of tumors in vivo (Alarcon et al, 1996). In light of these findings, we investigated the role of p53 in c-Myc-induced apoptosis under hypoxic conditions. We also determined the contribution of c-Myc activation and p53 to apoptosis induced by a genotoxic stress such as gamma irradiation. We report here that expression of antisense RNA, which is complimentary to the p53 mRNA, effectively delays the onset of apoptosis induced by c-Myc activation in cells exposed to low serum or hypoxic conditions. Furthermore, activation of c-Myc results in substantial sensitization of Rat-1 fibroblasts to radiationinduced apoptosis, which is also suppressed by antisense inhibition of p53 protein accumulation. 


\section{Results}

Rat-1MycER fibroblasts were generated that express a chimera of the murine c-Myc protein and a modified hormone binding domain of the estrogen receptor (c-MycER). Because expression of human dominant negative p53 alleles failed to alter p53 function in Rat-1 fibroblasts (data not shown), we transfected Rat-1MycER fibroblasts to express a full length antisense RNA complimentary to rodent p53 mRNA (Rat1MycER-ASp53). Rat-1MycER cells were also generated which overexpress the human $\mathrm{Bcl}-2$ protein (Rat-1MycER$\mathrm{Bcl}-2)$. The conditional c-MycER in these cells was activated by the addition of the estrogen analog 4-Hydroxytamoxifen (4HT). This compliment of cell lines (Table 1) along with parental Rat-1 fibroblasts and vector transfected control cells (Rat-1MycER-Neo and Rat-1MycER-Zeo) were used to study the role of p53 in mediating c-Myc-induced apoptosis under hypoxic conditions and apoptosis resulting from c-Myc activation and gamma irradiation.

Since activation of c-Myc in Rat-1MycER fibroblasts has been shown to result in rapid accumulation of the p53 protein in serum starved fibroblasts (Hermeking and Eick, 1994), p53 protein levels were assessed in Rat-1, Rat1MycER-ASp53 and control Rat-1MycER-Zeo cells at 0 and $24 \mathrm{~h}$ following c-Myc activation (Figure 1a). No change in the abundance of p53 was detected in Rat-1 cells which do not express the c-MycER chimera. However, p53 protein levels were increased by more than 8 fold in Rat-1MycERZeo fibroblasts $24 \mathrm{~h}$ after activation of c-Myc. In untreated Rat-1MycER-ASp53 cells, no detectable p53 protein was present, while cells treated for $24 \mathrm{~h}$ with $4 \mathrm{HT}$ accumulated p53 protein only to the level of untreated Rat-1 control cells. Thus, antisense RNA is able to downregulate p53 expression in Rat-1 fibroblasts. Although p53 accumulation induced by activated c-Myc is not completely eliminated in Rat-1MycER-ASp53 cells, it is substantially delayed relative to Rat-1MycER-Zeo control cells.

As an indicator of c-Myc activation in these cell lines, we examined protein levels of the cyclin dependent kinase inhibitor, p27 ${ }^{\mathrm{Kip}-1}$, following exposure to $4 \mathrm{HT}$. Recent studies have demonstrated that the abundance of p27 protein in serum starved Rat-1MycER fibroblasts decreases substantially following activation of c-Myc (Steiner et al, 1995). We examined p27 protein in cells which were serum starved in $0.1 \%$ FBS for $48 \mathrm{~h}$ in the presence or absence of $10^{-7} \mathrm{M}$
$4 \mathrm{HT}$ for the last $24 \mathrm{~h}$ of incubation (Figure 1b). As expected, no difference in p27 protein abundance was observed in Rat-1 fibroblasts which did not express the c-MycER chimera. However, all of the clones expressing c-MycER had a similar reduction in p27 protein levels. Thus, activation of the c-MycER chimera occurred in all of the cell lines in which it was expressed. These results also suggest that neither $\mathrm{Bcl}-2$ overexpression nor loss of normal p53 accumulation limits the ability of activated c-Myc to cause a reduction in p27 protein levels. This finding implies that $\mathrm{Bcl}-2$ overexpression or loss of p53 do not affect c-Myc function, but rather act downstream of c-Myc to suppress
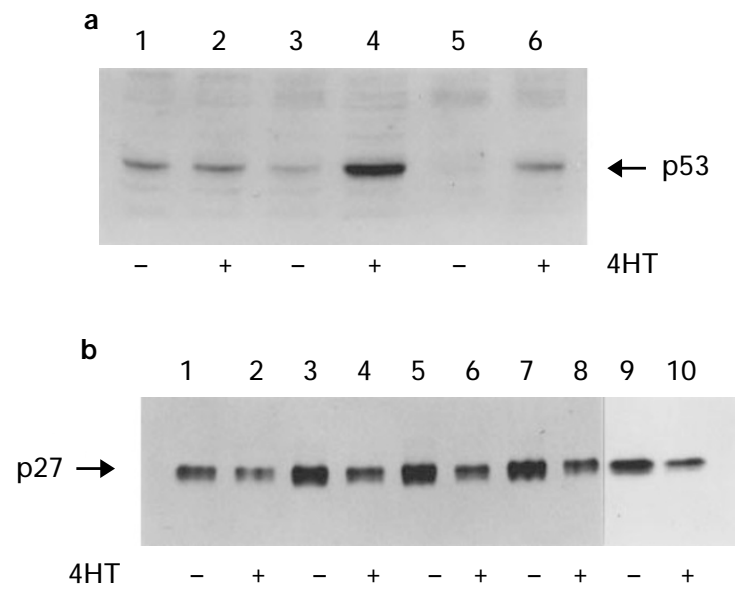

Figure 1 Immunoblot analysis of p53 and p27 protein levels in transfected Rat-1 fibroblasts (a) Effect of antisense p53 RNA expression on p53 protein abundance and accumulation following c-Myc activation. Immunodetection of p53 protein in Rat-1 (Lanes 1, 2), Rat-1MycER-Zeo (Lanes 3, 4) and Rat1MycER-ASp53 (Lanes 5,6 ) incubated in DMEM+0.1\% FBS for $24 \mathrm{~h}$ in the absence (Lanes $1,3,5$ ) or presence (Lanes $2,4,6)$ of $10^{-7} \mathrm{M} 4 \mathrm{HT} .40 \mu \mathrm{g}$ of each protein sample was resolved on a $10 \%$ polyacrylamide gel, transferred to nitrocellulose and rat p53 protein was detected with a sheep polyclonal antibody. (b) Effect of c-Myc activation on p27 protein abundance in serum starved fibroblasts. Immunodetection of p27 protein in Rat-1 (Lanes 1, 2), Rat1MycER (Lanes 3, 4), Rat-1MycER-Zeo (Lanes 5, 6) and Rat-1MycER-ASp53 (Lanes 7, 8) and Rat-1MycER-Bcl-2 cells (Lanes 9, 10) incubated in DMEM $+0.1 \%$ FBS for $24 \mathrm{~h}$ followed by an additional $24 \mathrm{~h}$ incubation in the absence (Lanes 1, 3, 5, 7, 9) or presence (Lanes 2, 4, 6, 8, 10) of $10^{-7} \mathrm{M} 4 \mathrm{HT}$. $20 \mu \mathrm{g}$ of each protein sample was resolved on a $12 \%$ polyacrylamide gel, transferred to nitrocellulose and rat p27 protein was detected with a rabbit monoclonal antibody

Table 1 Cell lines, transfected genes and responses to ionizing radiation

\begin{tabular}{|c|c|c|c|c|c|}
\hline \multirow[b]{2}{*}{ Cell name } & \multirow{2}{*}{$\begin{array}{c}\text { Exogenous } \\
\text { molecules expressed }\end{array}$} & \multicolumn{2}{|c|}{ Percent apoptotic, 5 Gy* } & \multicolumn{2}{|c|}{ Surviving fraction, 5 Gy $\dagger$} \\
\hline & & $-4 \mathrm{HT}$ (Myc OFF) & $+4 \mathrm{HT}$ (Myc ON) & $-4 \mathrm{HT}$ (Myc OFF) & $+4 \mathrm{HT}(\mathrm{Myc}$ ON) \\
\hline $\begin{array}{l}\text { Rat-1 } \\
\text { Rat-1 MycER } \\
\text { Rat-1 MycER-ASp53 } \\
\text { Rat-1 MycER-Zeo } \\
\text { Rat-1 MycER-Bcl-2 } \\
\text { Rat-1 MycER-Neo }\end{array}$ & $\begin{array}{c}\text { none } \\
\text { c-MycER } \\
\text { c-MycER, Antisense p53 RNA } \\
\text { c-MycER } \\
\text { c-MycER, Bcl-2 } \\
\text { c-MycER }\end{array}$ & $\begin{array}{r}6.4 \pm 1.2 \\
7.7 \pm 0.5 \\
5.8 \pm 0.6 \\
8.3 \pm 0.9 \\
5.0 \pm 0.2 \\
10.1 \pm 1.3\end{array}$ & $\begin{array}{r}7.5 \pm 0.9 \\
33.1 \pm 2.3 \\
10.6 \pm 0.2 \\
38.9 \pm 2.6 \\
5.4 \pm 0.4 \\
40.2 \pm 1.9\end{array}$ & $\begin{array}{c}0.204 \pm 0.036 \\
\text { N.D. } \\
0.175 \pm 0.014 \\
0.178 \pm 0.029 \\
0.221 \pm 0.018 \\
0.170 \pm 0.032\end{array}$ & $\begin{array}{l}0.216 \pm 0.034 \\
\text { N.D. } \\
0.097 \pm 0.013 \\
0.067 \pm 0.010 \\
0.213 \pm 0.034 \\
0.060 \pm 0.003\end{array}$ \\
\hline
\end{tabular}

*Mean percent apoptotic cells + standard deviation $24 \mathrm{~h}$ following 5 Gy gamma irradiation in the presence $\left(+4 \mathrm{HT}\right.$, Myc ON) or absence $\left(-4 \mathrm{HT}\right.$, Myc OFF) of $10^{-7} \mathrm{M}$ $4 \mathrm{HT}$. † Surviving fraction \pm standard deviation of cells after 5 Gy gamma irradiation in the presence $\left(+4 \mathrm{HT}\right.$, Myc ON) or absence $\left(-4 \mathrm{HT}\right.$, Myc OFF) of $10^{-7} \mathrm{M} 4 \mathrm{HT}$ as determined by clonogenic survival analysis. N.D. Surviving Fraction at 5 Gy not calculated for this cell line 
apoptosis. Interestingly, serum starved Rat-1 fibroblasts expressed somewhat lower levels of p27 protein than did the transfected cell lines which express c-MycER. The reason for this is not clear.

To determine the effects of c-Myc activation in low serum or hypoxic conditions or in conjunction with gamma irradiation in this system, bisbemzimide (Hoescht 33342) stained, Rat-1MycER nuclei were analyzed microscopically for morphologic features of apoptosis following these treatments in the presence or absence of 4HT (Figure 2). A large proportion of nuclei from serum starved Rat1 MycER cells exhibited the morphologic characteristics of apoptosis including chromatin condensation and nuclear fragmentation following $24 \mathrm{~h}$ incubation in the presence of $4 \mathrm{HT}$, whereas only a few apoptotic nuclei were observed in the absence of 4HT (Figure 2A and D). Microscopic examination of nuclei following exposure to hypoxia $(0.02 \%$ Oxygen) for a period of $36 \mathrm{~h}$ in the presence or absence of $4 \mathrm{HT}$ revealed similar results as those obtained under low serum conditions. Many Rat-1MycER cells incubated under hypoxic conditions in the presence of 4HT appeared apoptotic, whereas few apoptotic nuclei were observed in the absence of $4 \mathrm{HT}$ (Figure $2 \mathrm{~B}$ and $\mathrm{E}$ ). In gamma irradiated fibroblasts, c-MycER activation by 4HT resulted in substantially greater numbers of apoptotic nuclei than when c-MycER was not activated (Figure 2C and $F$ ). Micronuclei indicative of DNA damage following irradiation were observed regardless of the c-MycER activation status of the cells. These results demonstrate that c-Myc activation sensitizes Rat-1 fibroblasts to apoptosis induced by serum deprivation, hypoxia or gamma irradiation.

To quantitate c-Myc mediated apoptosis in serum deprived fibroblasts and to examine the requirement for p53 to execute apoptosis under these conditions, flow cytometric analysis of cellular DNA content was performed on Rat-1, Rat-1MycER, Rat-1MycER-ASp53 and Rat$1 \mathrm{MycER}-\mathrm{Bcl}-2$ cells following serum deprivation in the presence or absence of $4 \mathrm{HT}$ (Figure 3). Serum starved parental Rat-1 fibroblasts exhibited very little apoptosis in the presence or absence of $4 \mathrm{HT}$. However, more than $55 \%$ of Rat-1MycER and vector transfected Rat-1MycER cells underwent apoptosis in low serum within $24 \mathrm{~h}$ of c-Myc activation. This effect was more pronounced following $48 \mathrm{~h}$ of serum deprivation. In contrast, $\mathrm{Bcl}-2$ overexpressing cells were significantly less sensitive to apoptosis induced by $48 \mathrm{~h}$ of serum deprivation in the presence of $4 \mathrm{HT}$ than were vector transfected counterparts $(P<0.0001)$. Also, Rat1MycER-ASp53 cells expressing antisense RNA against p53 mRNA exhibited substantially less apoptosis than control cells following $24 \mathrm{~h}$ of c-Myc activation in low serum $(P=0.0007)$. However, unlike $\mathrm{Bcl}-2$ overexpressing cells, by $48 \mathrm{~h}$, many Rat-1MycER-ASp53 cells were dying by apoptosis. Thus, expression of antisense p53 RNA in Rat-1MycER-ASp53 cells effectively delayed apoptosis induced by c-Myc activation under low serum conditions and is consistent with the observation that following $24 \mathrm{~h}$ of c-Myc activation, Rat-1MycER-ASp53 cells had accumulated p53 protein only to the level of untreated vector transfected control cells. Taken together these results
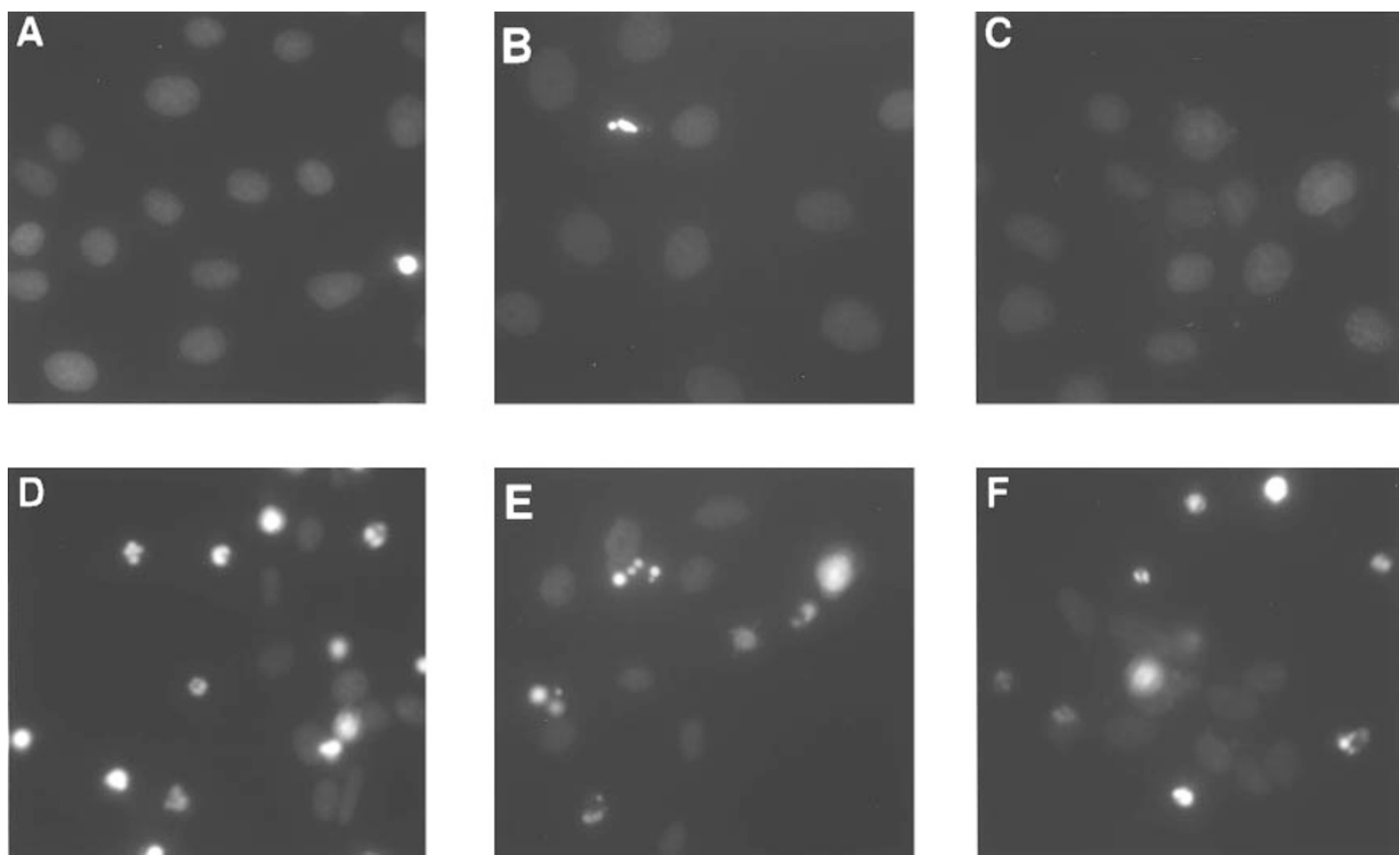

Figure 2 Effect of c-MycER activation on apoptosis of serum starved, hypoxic or gamma irradiated Rat- 1 fibroblasts. $5 \times 10^{5}$ Rat-1-MycER cells in $60 \mathrm{~mm}$ tissue culture dishes were incubated in the absence (panels $\mathbf{A}, \mathbf{B}$ and $\mathbf{C}$ ) or presence (panels $\mathbf{D}, \mathbf{E}$ and $\mathbf{F}$ ) of $10^{-7} \mathrm{M} 4 \mathrm{HT}$ and under low serum conditions ( $0.1 \% \mathrm{FBS}$; panel $\mathbf{A}$ and $\mathbf{D}$ ), hypoxic conditions ( $0.02 \%$ Oxygen; panels $\mathbf{B}$ and $\mathbf{E}$ ) or following gamma irradiation ( $5 \mathrm{~Gy}$; panels $\mathbf{C}$ and $\mathbf{F}$ ). After $24 \mathrm{~h}$ of serum deprivation or $36 \mathrm{~h}$ of hypoxia or $24 \mathrm{~h}$ following irradiation, Hoescht 33342 was added to culture dishes $(5 \mu \mathrm{g} / \mathrm{ml}$ final concentration) and nuclei were photographed under UV illumination at $600 \times$ magnification 
suggest that p53 accumulation above levels of untreated, exponentially growing cells is required to execute apoptosis induced by c-Myc activation in serum starved fibroblasts.

The requirement for p53 in c-Myc-induced apoptosis under hypoxic conditions was next examined using flow cytometric DNA content analysis (Figure 4). Hypoxia alone for up to $48 \mathrm{~h}$ without c-Myc activation (parental Rat-1 cells) induced very few cells to undergo apoptosis. Twenty-four hours after c-Myc activation under hypoxic conditions, apoptosis was apparent in 15\% of Rat-1MycER and vector transfected Rat-1MycER-Zeo and Rat-1MycER-Neo cells. Longer exposures of Rat-1MycER and vector transfected controls cells to hypoxia in the presence of $4 \mathrm{HT}$ resulted in a substantial increase in apoptosis. Apoptosis induced by low oxygen conditions was suppressed by $\mathrm{Bcl}-2$ overexpression and antisense p53 RNA expression. Bcl-2 overexpressing Rat-1MycER-Bcl-2 cells were almost completely resistant to $\mathrm{c}$-Myc-induced apoptosis under hypoxic conditions for as long as $48 \mathrm{~h}(P=0.0002$ compared to Rat1MycER-Neo control cells). Rat-1MycER-ASp53 cells were also relatively resistant to hypoxia-induced apoptosis for up to $36 \mathrm{~h}$ of exposure to $4 \mathrm{HT}$ under hypoxic conditions ( $P=0.0009$ compared to Rat-1MycER-Zeo cells). However, the percentage of apoptotic cells increased sharply between 36 and $48 \mathrm{~h}$ incubation in the same conditions. Under hypoxic conditions, apoptosis is substantially delayed in Rat-1MycER-ASp53 cells relative to controls. This is also consistent with the delayed accumulation of

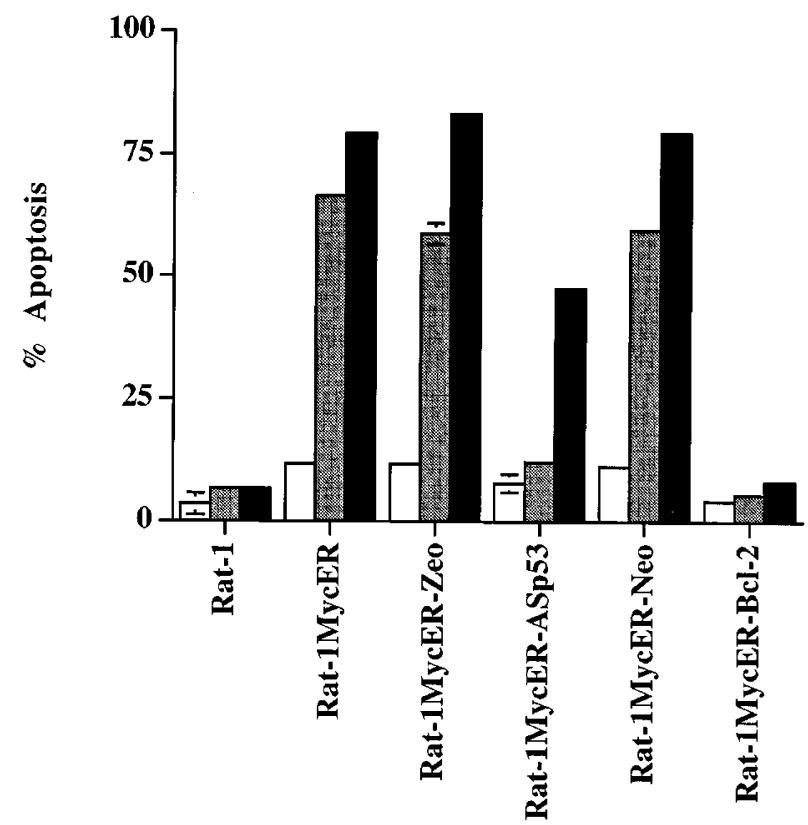

Figure 3 Effect of antisense p53 and Bcl-2 expression on c-Myc-induced apoptosis in low serum conditions. Cultures treated for $48 \mathrm{~h}$ in DMEM+0.1\% FBS in the absence of $4 \mathrm{HT}$ (open bars), for $24 \mathrm{~h}$ in DMEM+0.1\% FBS $+10^{-7} \mathrm{M}$ $4 \mathrm{HT}$ (gray bars) or for $48 \mathrm{~h}$ in DMEM $+0.1 \% \mathrm{FBS}+10^{-7} \mathrm{M} 4 \mathrm{HT}$ (black bars) were harvested and analyzed for DNA content by PI staining and flow cytometry. The percent apoptosis represents the fraction of cells exhibiting sub-diploid DNA content. Error bars represent one standard deviation from the mean of four experiments. Error bars are not shown for bars with standard deviation less than $2 \%$ p53 protein following c-Myc activation, indicating that p53 mediates c-Myc-induced apoptosis under hypoxic as well as low serum conditions.

Next, experiments were performed to determine the contribution of activated c-Myc and p53 accumulation to apoptosis induced by ionizing radiation. Exponentially growing cells were incubated in DMEM containing $10 \%$ FBS in the presence or absence of $4 \mathrm{HT}$ for $4 \mathrm{~h}$ prior to treatment with 5 Gy of gamma radiation. Previous studies have demonstrated that biochemical events resulting from c-MycER activation occur within $4 \mathrm{~h}$ of $4 \mathrm{HT}$ treatment (Hermeking and Eick, 1994). Thus, c-Myc activity is deregulated in these cells at the time of irradiation. Quantitation of apoptosis at 24 and $36 \mathrm{~h}$ following irradiation revealed that in the absence of c-Myc activation, 5 Gy of gamma radiation induced approximately $10 \%$ of the cells from any of the fibroblast lines studied to undergo apoptosis (Figure $5 \mathrm{a}$ and b). However, in Rat1 MycER cells and vector transfected Rat-1MycER cells irradiated following c-Myc activation, $30-40 \%$ of the cell population underwent apoptosis within $24 \mathrm{~h}$. In irradiated cells overexpressing the human $\mathrm{Bcl}-2$ protein there was no difference in the number of apoptotic cells in cultures treated with $4 \mathrm{HT}$ prior to irradiation compared to cultures without c-Myc activation. The differences in apoptosis between Rat-1, vector transfected Rat-1MycER and Rat-

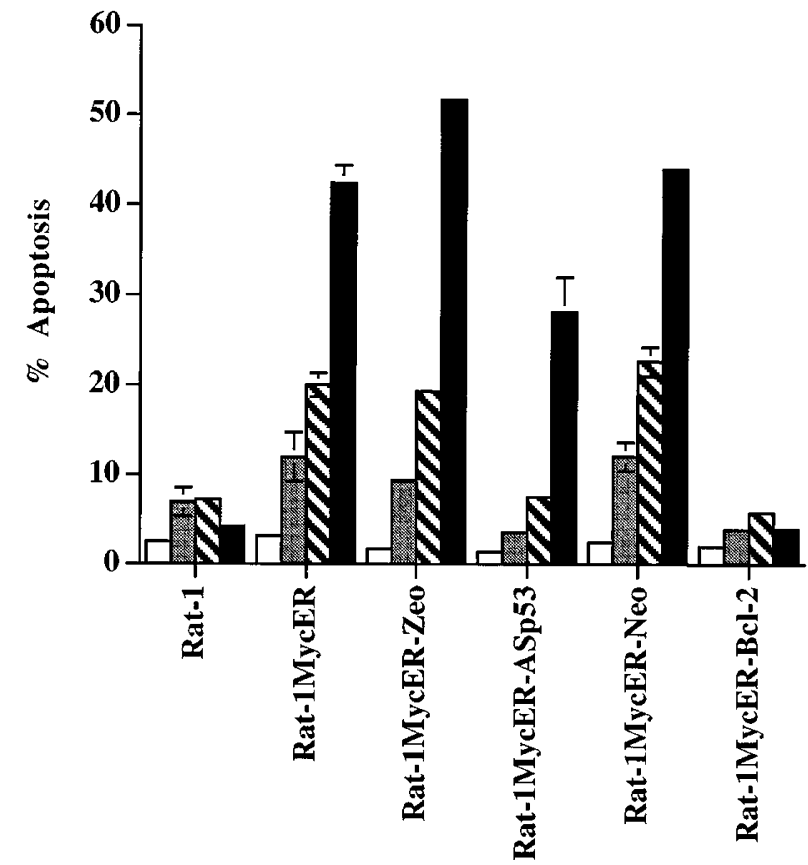

Figure 4 Effect of antisense p53 and Bcl-2 expression on c-Myc-induced apoptosis under hypoxic conditions. Cultures treated for $24 \mathrm{~h}$ in air in the absence of $4 \mathrm{HT}$ (open bars), for $24 \mathrm{~h}$ under hypoxic conditions in the presence of $10^{-7} \mathrm{M} 4 \mathrm{HT}$ (gray bars), for $36 \mathrm{~h}$ under hypoxic conditions in the presence of $10^{-7} \mathrm{M} 4 \mathrm{HT}$ (hatched bars) or for $48 \mathrm{~h}$ under hypoxic conditions in the presence of $10^{-7} \mathrm{M} 4 \mathrm{HT}$ (black bars) were harvested and analyzed for DNA content by PI staining and flow cytometry. The percent apoptosis represents the fraction of cells exhibiting sub-diploid DNA content. Error bars represent one standard deviation from the mean of four experiments. Error bars are not shown for bars with standard deviation less than $2 \%$ 

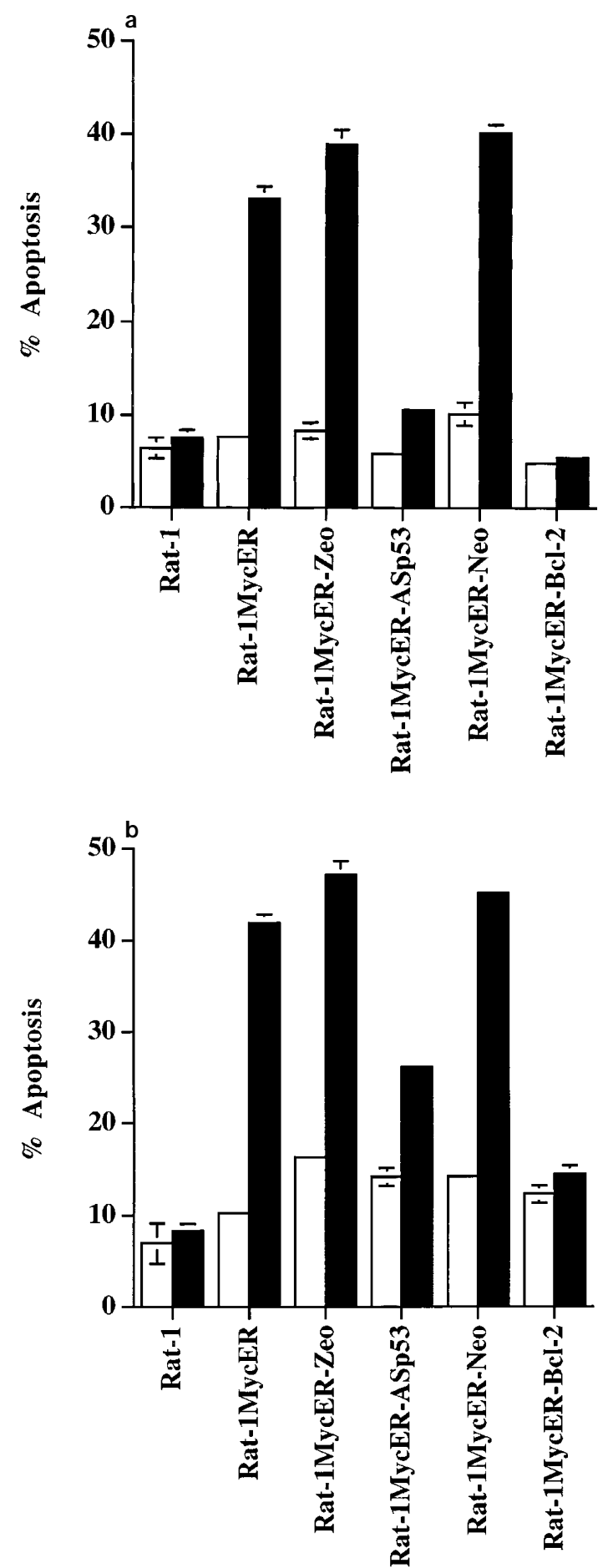

Figure 5 Effect of antisense p53 and $\mathrm{Bcl}-2$ expression on apoptosis induced by a combination of c-Myc activation and gamma radiation. (a) Cultures were harvested and analyzed for DNA content by PI staining and flow cytometry $24 \mathrm{~h}$ after $5 \mathrm{~Gy}$ irradiation in the absence (open bars) or presence (black bars) of $10^{-7} \mathrm{M} 4 \mathrm{HT}$. (b) Cultures were harvested and analyzed for DNA content by PI staining and flow cytometry $36 \mathrm{~h}$ after $5 \mathrm{~Gy}$ irradiation in the absence (open bars) or presence (black bars) of $10^{-7} \mathrm{M} 4 \mathrm{HT}$. The percent apoptosis represents the fraction of cells exhibiting sub-diploid DNA content. Error bars represent one standard deviation from the mean of four experiments. Error bars are not shown for bars with standard deviation less than $2 \%$
$1 \mathrm{MycER}-\mathrm{Bcl}-2$ cells was reflected in clonogenic survival assays (Table 1). The surviving fraction of Rat-1MycER$\mathrm{Neo}$ and Rat-1MycER-Zeo cells following 5 Gy gamma irradiation was significantly reduced in the presence of $4 \mathrm{HT}$ as compared to identical cells irradiated in the absence of $4 \mathrm{HT}$ ( $<0.005$ and $P<0.001$ respectively). Furthermore, the presence of $4 \mathrm{HT}$ did not significantly alter the clonogenic survival of parental Rat-1 $(P>0.5)$ or Rat-1MycER-Bcl-2 $(P>0.5)$ cells. These results suggest that c-Myc-mediated alterations in radiation-induced apoptosis can affect overall radiosensitivity as measured by clonogenic survival. Rat1MycER-ASp53 cells were also substantially more resistant to apoptosis $24 \mathrm{~h}$ following c-Myc activation and irradiation compared to Rat-1MycER and vector transfected controls $(P=0.0006)$. However, unlike Rat-1MycER-Bcl-2 cells, the number of apoptotic Rat-1MycER-ASp53 cells increased slightly in cultures exposed to $4 \mathrm{HT}$ prior to irradiation as compared to cells cultured without $4 \mathrm{HT}$. This effect was more pronounced at $36 \mathrm{~h}$ than at $24 \mathrm{~h}$ following irradiation. Clonogenic survival analysis of Rat-1MycER-ASp53 cells revealed that these cells were more radiosensitive $(P<0.001)$ in the presence of $4 \mathrm{HT}$ than in its absence (Table 1). This result is consistent with the observation that antisense p53 RNA expression delays but does not eliminate p53 accumulation and apoptosis in cells with deregulated c-Myc activity. These results suggest that fibroblasts with activated c-Myc were considerably more sensitive to radiation-induced apoptosis than fibroblasts without activated c-Myc. Furthermore, apoptosis induced by c-Myc activation and gamma irradiation is inhibited by overexpression of $\mathrm{Bcl}-2$ or inhibition of p53 accumulation via antisense RNA expression.

\section{Discussion}

This study provides evidence that p53 accumulation is important for the execution of apoptosis in serum starved, hypoxic or gamma irradiated cells with deregulated c-Myc activity. p53 appears to be an important mediator of apoptosis in a variety of cells transformed by many means, however, the apoptotic potential of cells which have not been oncogenically transformed is quite low. We show here that manipulation of the activity of a single oncogene (c-Myc) results in significant sensitization of fibroblasts to apoptosis induced by a variety of physical and environmental stresses and that this approach requires p53 accumulation. Inhibition of p53 accumulation inhibited both hypoxia and radiation-induced apoptosis in the absence of c-Myc activation albeit a relatively minimal effect. On the other hand, in cells with active c-Myc, the effects of antisense RNA mediated delay in p53 accumulation were profound. Thus, while p53 appears to modulate the sensitivity of these cells to stress-induced apoptosis, its effects are most pronounced in oncogenically altered cells.

At present, it is unclear how or why c-Myc activation induces apoptosis in serum starved, hypoxic or irradiated fibroblasts. Two models have been proposed to explain cMyc-induced apoptosis of serum deprived fibroblasts (Harrington et al, 1994a). The 'conflict' model suggests that activated c-Myc provides a proliferation signal and that serum deprivation provides a growth arrest signal. Upon 
receiving these conflicting messages the cell enters the cell death pathway and undergoes apoptosis unless a genetic inhibitor of the cell death program (i.e. Bcl-2) is present. According to the second model, the 'dual signal' model, cMyc activation initiates both the proliferation and the cell death pathways simultaneously. In this model, serum derived 'survival' cytokines or genetic inhibitors of the cell death machinery are required to suppress apoptosis and allow proliferation to occur. Evidence in support of the 'dual signal' model comes from experiments that have shown that c-Myc activation induced apoptosis of fibroblasts arrested beyond the stage of the cell cycle which requires growth factors for proliferation (Harrington et al., 1994b). In addition, specific cytokines including Insulin-like Growth Factor-1 (IGF-1) and Platelet Derived Growth Factor (PDGF) are able to inhibit c-Myc-induced apoptosis of serum deprived Rat-1 fibroblasts, while other cytokines such as Epidermal Growth Factor (EGF) and basic Fibroblast Growth Factor (bFGF) are unable to suppress c-Myc-induced apoptosis, even at concentrations tenfold greater than those commonly used when these factors are used as mitogens (Harrington et al, 1994b).

The dual signal model, however, does not explain how cMyc activation under hypoxic conditions or in combination with gamma irradiation induces apoptosis of fibroblasts cultured in media containing the necessary to cytokines to suppress c-Myc-induced apoptosis of normal fibroblasts under aerobic conditions. It is possible that growth arrest resulting from DNA damage or exposure to hypoxic conditions is capable of inducing apoptosis of cells with activated c-Myc by a mechanism resembling the 'conflict' model. Alternatively, both hypoxia and gamma irradiation can alter the expression of a variety of genes (Fandrey, 1995; Fornace, 1992). Induced or repressed expression of certain stress response genes might in some way block or remove the inhibition of the c-Myc-initiated cell death program in a manner analogous to removal of serum derived 'survival' factors. Such a situation would be consistent with the 'dual signal' model of c-Myc-induced apoptosis. Regardless of whether c-Myc-induced apoptosis occurs from conflicting growth signals or loss of repression of a c-Myc-initiated death pathway, p53 appears to be an important mediator c-Myc-induced cell death under environmental stresses which affect tumor growth, evolution and response to therapy.

\section{Materials and Methods}

\section{Cell lines and transfection}

Rat-1MycER fibroblasts were generated by transfecting parental Rat-1 fibroblasts with the pBpuro-c-MycER plasmid as previously described (Graeber et al, 1996). These cells express the c-MycER fusion protein that is activated by addition of 4-Hydroxytamoxifen (4HT) to the media of cultured cells at a concentration of $10^{-7} \mathrm{M}$ (Littlewood et al, 1995). Rat-1MycER clone YY8ME4 was used for all experiments described in this report.

Rat-1MycER clone YY8ME4 was subsequently transfected with the pZeoSV-ASp53 plasmid which expresses an antisense RNA complimentary to the full length mouse p53 mRNA. pZeoSV-ASp53 was constructed by directional ligation of the Xhol/Xbal fragment of the pCMVp53wt plasmid (Eliyahu et al, 1989) which contains the wild-type mouse p53 cDNA into Spel/Xhol digested pZeoSV expression plasmid (Invitrogen; San Diego, CA) in the antisense orientation. pZeoSV-ASp53 or the empty pZeoSV vector, were transfected into Rat-1MycER cells using the Lipofectamine transfection reagent according to the manufacturers protocol (Gibco BRL; Bethesda, MD). Transfected cells were selected in growth media containing $350 \mu \mathrm{g} / \mathrm{ml}$ Zeocin (Invitrogen; San Diego, CA). Four stable, pZeoSV transfected clones were pooled and this pool is referred to as Rat-1MycER-Zeo throughout this report. Stable, Zeocin resistant, pZeoSV-ASp53 transfected clones were analyzed by immunoblotting for loss of p53 protein expression as described below. One clone, Rat-1MycER-ASp53 was identified which had no detectable p53 protein expression.

Rat-1MycER (clone YY8ME4) cells were also infected with the cosMSV-tk-Neo-hBcl-2 retroviral vector which expresses human Bcl2 protein or the empty cosMSV-tk-Neo vector as previously described (Naumovski and Cleary, 1994). Retrovirus infected Rat1 MycER cells were selected in $600 \mu \mathrm{g} / \mathrm{ml} \mathrm{G418} \mathrm{(Geneticin,} \mathrm{Gibco}$ $\mathrm{BRL}$, Bethesda, MD). Expression of human Bcl-2 protein was determined by immunoblotting. Three $\mathrm{G} 418$ resistant $\mathrm{Bcl}-2$ expressing clones were pooled and is referred to as Rat-1MycER$\mathrm{Bcl}-2$. Four G418 resistant clones transfected with the empty retroviral vector were pooled for use as vector transfected controls (Rat-1MycER-Neo).

All cells were maintained in Dulbecco's Modified Eagle's Medium (DMEM) containing 10\% FBS and no selection antibiotics (Rat-1), $2.5 \mu \mathrm{g} / \mathrm{ml}$ puromycin (Rat-1MycER), $2.5 \mu \mathrm{g} / \mathrm{ml}$ puromycin and $300 \mu \mathrm{g} /$ ml G418 (Rat-1MycER-Bcl-2, Rat-1MycER-Neo), or $2.5 \mu \mathrm{g} / \mathrm{ml}$ puromycin and $250 \mu \mathrm{g} / \mathrm{ml}$ Zeocin (Rat-1MycER-ASp53, Rat-1MycER-Zeo). Selection antibiotics were withheld from cultures for at least 5 days prior to the start of experiments.

\section{Immunoblotting}

Following treatment as described above, adherent cells in monolayer cultures were harvested by trypsinization and cell pellets were solubilized in $200 \mu$ l loading buffer (100 mM Tris pH 8.8, 4\% (w/v) SDS, $5 \mathrm{mM}$ EDTA, $15 \%$ (w/v) sucrose, $10 \mathrm{mM}$ dithiothreitol, $0.01 \%$ bromophenol blue). Protein concentrations for each sample were determined using the D/C protein assay kit (BioRad, Hercules, CA) according to manufacturers specifications. Equivalent amounts of protein (25 $\mu \mathrm{g}$ for p27 and $40 \mu \mathrm{g}$ for p53 gels) were run on SDSPAGE gels (12\% for p27 gels, $10 \%$ for p53 gels). Proteins were transferred to nitrocellulose membranes and specific proteins were detected using standard immunoblotting procedures. p53 protein was detected with sheep anti-p53 polyclonal antisera, AB-7 (Oncogene Research Products, Calbiochem, Cambridge, MA) and p27 with a rabbit anti-mouse p27 monoclonal antibody, C-19 (Santa Cruz Biotechnology, Santa Cruz, CA). Positively staining protein bands were detected with the ECL Enhanced Chemiluminescence detection system according to manufacturers protocol (Amersham Life Science, Arlington Heights, IL).

\section{Apoptosis quantitation}

Exponentially growing cell cultures were incubated in DMEM in either $10 \%$ of $0.1 \% \mathrm{FBS}$ and in the presence or absence of $10^{-7} \mathrm{M} 4 \mathrm{HT}$ as indicated. Aerobic cultures were performed at $37^{\circ} \mathrm{C}$ in air containing $5 \% \mathrm{CO}_{2}$. Hypoxic incubations were performed in DMEM containing $10 \%$ FBS in an anaerobic incubator as previously described (Graeber 
et al, 1996). For all hypoxic incubations the oxygen concentration was $0.02 \%$ and was carried out for the indicated times in the presence or absence of $10^{-7} \mathrm{M} 4 \mathrm{HT}$. Gamma irradiation was performed following $4 \mathrm{~h}$ of incubation in DMEM containing $10 \%$ FBS in the presence or absence of $10^{-7} \mathrm{M} 4 \mathrm{HT}$. Cells were irradiated with a dose of $5 \mathrm{~Gy}$ using a Cesium-137 source. Apoptosis was analyzed at various times following these treatments by two different methods. First, bisbenzimide (Hoescht 33342) was added directly to the culture medium at a final concentration of $5 \mu \mathrm{g} / \mathrm{ml}$. Cells were then visualized microscopically under UV illumination and apoptotic nuclei were scored based on chromatin condensation and nuclear morphology. Photomicrographs were taken at $600 \times$ magnification. Secondly, apoptosis was quantitated by flow cytometric DNA content analysis using a modification of the methods described by Nicolletti et al (1991) and Larsen (1994). Briefly, cultures were harvested and cells pelleted by centrifugation, washed twice in calcium and magnesium free Dulbecco's Phosphate Buffered Saline (D-PBS), resuspended in icecold freezing buffer $(250 \mathrm{mM}$ sucrose; $5 \%(\mathrm{v} / \mathrm{v})$ dimethyl sulfoxide; $40 \mathrm{mM}$ sodium citrate $\mathrm{pH} 7.6$ ) and stored at $-70^{\circ} \mathrm{C}$. Prior to flow cytometry, frozen cell suspensions were thawed at room temperature, washed twice in calcium and magnesium free D-PBS and resuspended in $0.2 \mathrm{ml} \mathrm{D-PBS}$. An additional $0.2 \mathrm{ml} \mathrm{D-PBS}$ containing $0.5 \%(\mathrm{v} / \mathrm{v})$ Nonidet NP40, $20 \mu \mathrm{g} / \mathrm{ml}$ Propidium lodide (PI) and $0.2 \mathrm{mg} /$ $\mathrm{ml}$ RNAseA was then added to cell suspensions. Cell suspensions were gently shaken on ice for at least $1 \mathrm{~h}$ to follow permeabilization and DNA staining. Stained cells were analyzed on a Facstar flow cytometer (Becton Dickenson, San Jose, CA) for DNA content. Cells containing sub-diploid DNA content were considered apoptotic. The validity of the flow cytometric technique was confirmed by sorting cell populations into DNA less than $2 \mathrm{~N}$ and DNA greater than or equal to $2 \mathrm{~N}$. Microscopic analysis of these two populations confirmed that all subdiploid cells exhibited apoptotic morphology while very few cells $(<5 \%)$ with $2 \mathrm{~N}-4 \mathrm{~N}$ DNA content appeared apoptotic. All data points represent the mean \pm standard deviation of at least three independent experiments. P-values were determined using a two-tailed Student's t-test.

\section{Clonogenic survival analysis}

Colony assays were performed on Rat-1, Rat-1MycER-Zeo, Rat1MycER-ASp53, Rat-1MycER-Neo and Rat-1MycER-Bcl-2 cells by standard methods. Briefly, feeder layers were prepared by plating 300000 lethally irradiated cells (60 Gy) on $100 \mathrm{~mm}$ tissue culture plates and incubating at $37^{\circ} \mathrm{C}$ for $4 \mathrm{~h}$ to allow feeder layers to adhere. 100 or 500 cells (for unirradiated and irradiated plates respectively) were plated over feeder layer cells and allowed to adhere. After plating, growth media was changed to fresh media containing 0 or $10^{-7} \mathrm{M} 4 \mathrm{HT}$ and cells were incubated an additional $4 \mathrm{~h}$ to activate the c-MycER chimera. After incubation, plated cells were irradiated with 0 or 5 Gy using a Cesium-137 source. Plates were incubated $7-10$ days to allow colonies to form. Colonies were stained with $0.25 \%$ crystal violet in $70 \%$ ethanol and colonies containing greater than 30 cells were counted macroscopically. Surviving fractions and their standard deviations were calculated for each cell line. $P$ values were determined using a two-tailed Student's $t$-test.

\section{Acknowledgements}

The authors would like to thank L. Naumovski and M. Cleary for providing cosMSV-tk-Neo and cosMSV-tk-Neo-hBcl-2 retroviral constructs and T.G. Graeber for technical assistance and helpful discussions. This work was supported by a Biomedical Research Support Grant (S.J.K.), NIH grant CA68149-01 (S.J.K.), a Howard Hughes Medical Institute Young Investigator Award (A.J.G.), NIH grant CA64489-01 (A.J.G.) and NIH grant CA67166-01 (A.J.G.). B.A. Rupnow is a Howard Hughes Medical Institute Predoctoral Fellow.

\section{References}

Alarcon RM, Rupnow BA, Graeber TG, Knox SJ and Giaccia AJ (1996) Modulation of c-Myc activity and apoptosis in vivo. Can. Res. 56: 4315-4319

Bissonnette RP, Echeverri F, Mahboubi A and Green DR (1992) Apoptotic cell death induced by c-myc is inhibited by bcl-2. Nature 359: $552-554$

Eliyahu D, Michalovitz D, Eliyahu S, Pinhasi-Kimhi O and Oren M (1989) Wild-type p53 can inhibit oncogene-mediated focus formation. Proc. Natl. Acad. Sci. U.S.A. $86: 8763-8767$

Evan GI, Wyllie AH, Gilbert CS, Littlewood TD, L and H, Brooks M, Waters CM, Penn LZand Hancock DD (1992) Induction of apoptosis in fibroblasts by c-myc protein. Cell 69: $119-128$

Fandrey J (1995) Hypoxia-inducible gene expression. Respir. Physiol. 101: 1-10

Fanidi A, Harrington EA and Evan GI (1992) Cooperative interaction between c-myc and bcl-2-proto-oncogenes. Nature 359: $554-556$

Fornace AJJ (1992) Mammalian genes induced by radiation; activation of genes associated with growth control. Annu. Rev. Genet. 26: 507-526

Graeber TG, Osmanian C, Jacks T, Housman DE, Koch CJ, Lowe SW and Giaccia AJ (1996) Hypoxia-mediated selection of cells with diminished apoptotic potential in solid tumours. Nature 379: 88-91

Harrington EA, Bennett MR, Fanidi A and Evan GI (1994b) c-Myc-induced apoptosis in fibroblasts is inhibited by specific cytokines. EMBO J. 13: 3286-3295

Harrington EA, Fanidi A and Evan GI (1994a) Oncogenes and cell death. Curr. Opin. Genet. Dev. 4: 120-129

Hermeking H, Eick D (1994) Mediation of c-Myc-induced apoptosis by p53. Science 265: $2091-2093$

Larsen JK (1994) 'Washless' procedures for nuclear antigen detection. Methods Cell Biol. 41: 377-388

Littlewood TD, Hancock DC, Danielian PS, Parker MG, Evan GI (1995) A modified oestrogen receptor ligand-binding domain as an improved switch for the regulation of heterologous proteins. Nucleic Acids Res. 23: 1686-1690

Lowe SW (1995) Cancer therapy and p53. Curr. Opin. Oncol. 7: 547-553

Meichle A, Philipp A, Eilers M(1992) The functions of Myc proteins. Biochim. Biophys. Acta 1114: 129-146

Naumovski L, Cleary ML (1994) Bcl2 inhibits apoptosis associated with terminal differentiation of HL-60 myeloid leukemia cells. Blood 83: 2261-2267

Nicoletti I, Migliorati G, Pagliacci MC, Grignani F, Riccardi C (1991) A rapid and simple method for measuring thymocyte apoptosis by propidium iodide staining and flow cytometry. J. Immunol. Methods 139: 271-279

Steiner P, Philipp A, Lukas J, Godden-Kent D, Pagano M, Mittnacht S, Bartek J, Eilers $M$ (1995) Identification of a Myc-dependent step during the formation of active G1 cyclin-cdk complexes. EMBO J. 14: 4814-4826

Strasser A, Harris AW, Bath ML, Cory S (1990) Novel primitive lymphoid tumours induced in transgenic mice by cooperation between myc and bcl-2. Nature 348 : $331-333$

Wagner AJ, Kokontis JM, Hay N (1994) Myc-mediated apoptosis requires wild-type p53 in a manner independent of cell cycle arrest and the ability of p53 to induced p21waf1/cip1. Genes Dev. 8: 2817-2830 